\title{
Diseño y validación de un cuestionario para conocer las principales barreras y beneficios percibidos en el arbitraje femenino en el fútbol Design and validation of a questionnaire to identify the main barriers and perceived benefits of women's refereeing in football
}

*Francisco Segado Segado, *Juan Antonio Sánchez-Sáez, *María José Maciá Andreu, **Javier Sánchez-Sánchez, *Ana María Gallardo Guerrero

*Universidad Católica San Antonio de Murcia (España), **Universidad Europea de Madrid (España)

Resumen: Las mujeres han ido incorporándose poco a poco a la industria del fútbol durante los últimos años, no solo como deportista, sino también en otros estamentos de este sector como la dirección o el arbitraje. El objetivo del presente trabajo es diseñar y validar una herramienta de evaluación de las barreras percibidas en el arbitraje femenino, tanto durante la formación de las árbitras (de manera previa) como en su llegada al máximo nivel. Un total de 380 árbitras de ámbito nacional y territorial fueron consultadas a través de un cuestionario elaborado ad-hoc. Tras el análisis factorial confirmatorio de segundo orden, se establecieron 3 dimensiones: Percepción del Apoyo Institucional, Beneficios del Arbitraje y Percepción del Clima Social y Familiar. Tras el análisis de los resultados se puede concluir que se ha obtenido una herramienta válida y fiable para determinar las principales barreras asociadas al arbitraje femenino.

Palabras clave: Fútbol. Arbitraje. Mujeres. Barreras Percibidas.

Abstract: Women have been gradually joining the football industry in recent years, not only as sportswomen, but also in other areas of the sector such as management and refereeing. The aim of this work is to design and validate a tool for evaluating the barriers perceived in female refereeing, both during the training of female referees (in advance) and once they reach this level, as well as the analysis of the results obtained within the national territory. A total of 380 women referees at national and territorial level were consulted by means of a questionnaire developed on an ad hoc basis. After the second order confirmatory factor analysis, three dimensions were established: Perception of Institutional Support, Benefits of Refereeing and Perception of the Social and Family Climate. After the analysis of the results, it can be concluded that a valid and reliable tool has been obtained to determine the main barriers associated with female refereeing.

Keywords: Soccer. Refereeing. Women. Perceived Barriers.

\section{Introducción}

Aun hoy en día se detectan prácticas excluyentes dirigidas a las mujeres en el fútbol masculino (Cleland, Pope \& Williams, 2020), si bien depende de la cultura del país de referencia. Por ejemplo, en Estados Unidos, la alta participación de las jóvenes deportistas en la cultura futbolística promueve una cultura de género neutral (Flanagan, 2013), hecho que no ocurre en otros países donde las jugadoras no se sienten bienvenidas (Muller, 2014). Sin embargo, el incremento de la presencia de mujeres en roles diferentes al de deportistas, confirma que las estrategias planteadas están generando más oportunidades para las mujeres tanto en el ámbito deportivo como en la sociedad en general (Jones \& Edwards, 2013), mostrando no solo el talento femenino como ju-

Fecha recepción: 05-05-21. Fecha de aceptación: 15-07-21

Javier Sánchez-Sánchez

javier.sanchez2@universidadeuropea.es gadoras, sino también como árbitras (Reid \& Dallaire, 2019).

Tanto los gerentes como los medios de comunicación, han cuestionado en el pasado la competencia e idoneidad de las árbitras en las ligas profesionales masculinas, desencadenando un generalizado debate tanto en el ámbito del fútbol como en relación al público en general (Forbes, Edwards \& Fleming, 2015). Por ello, a pesar del incremento de la presencia de las árbitras y entrenadoras, estas consideran que en ocasiones no se les tiene en cuenta (Forbes, Edwards \& Fleming, 2015) y que las funciones de arbitraje se siguen considerando como masculinas (Reid \& Dallaire, 2019).

En la literatura existente, se plantean diferentes dimensiones de las barreras percibidas por las árbitras en su desempeño como árbitras de fútbol (Amorose, 2007; Cuskelly \& Hoye, 2013; Hong, Jeong \& Downward, 2019; Kim, 2017; Kim \& Hong, 2016; Logan, 2012; Mitchell, Gagné, Beaudry \& Dyer, 2012; Ridinger, Warner, Tingle \& Kim, 2017; Seibert, Wang \& Courtright, 2011; Tingle, Warner \& Sartore-baldwin, 
2014; Wu \& Liu, 2014). Estas barreras vienen marcadas principalmente por cuatro aspectos: sociales (mayor grado de exigencia o críticas negativas constantes), familiares (dificultades en la conciliación), institucionales (valoración del desempeño) o la propia motivación personal (sentirse bien consigo misma o tener un autoconcepto positivo) (Amorose, 2007; Cuskelly \& Hoye, 2013; Hong, Jeong \& Downward, 2019; Kim, 2017; Kim \& Hong, 2016; Logan, 2012; Mitchell, Gagné, Beaudry \& Dyer, 2012; Ridinger, Warner, Tingle \& Kim, 2017; Seibert, Wang \& Courtright, 2011;Tingle, Warner \& Sartore-baldwin, 2014; Wu \& Liu, 2014).

Respecto a la dimensión relacionada con el ámbito social, diferentes estudios señalan el sentimiento de incompetencia por parte de la sociedad hacia su labor (Forbes, Edwards \& Fleming, 2015; Theberge, 1990). En este sentido, para ser reconocidas como legítimas participantes y miembros de la comunidad del fútbol, tienen que luchar constantemente contra los estereotipos de género (Caudwell, 2011; Jones, 2008; Pope, 2013), siendo en determinados momentos incluso cuestionadas antes de entrar al campo de juego (Forbes, Edwards \& Fleming, 2015) y sintiéndose obligadas a demostrar su capacidad bajo la mirada atenta de los jugadores, entrenadores y espectadores para (re)afirmar su capacidad (Reid \& Dallaire, 2020).

Esto hace que algunas de las que experimentaron problemas o experiencias de género más evidentes no hayan continuado como árbitras, siendo uno de los motivos el abuso de género (Tingle, Warner \& SartoreBaldwin, 2014). Por contra, estudios realizados por Alonso-Arbiol, Arratibel y Gómez (2008) y Nordstrom, Warner y Barnes (2016), afirman que las colegiadas aseguran no haber vivido nunca una situación complicada o comprometida dentro de un terreno de juego, ni haber percibido diferencias de género importantes.

Por otro lado, investigaciones sobre la importancia de la pertenencia a una comunidad creada y desarrollada alrededor del arbitraje, resaltan que ésta fue fundamental para alcanzar experiencias positivas por parte de las árbitras (Kellett, Shilbury, 2007; Kellett \&Warner, 2011; Tingle, Warner \& Sartore-Baldwin, 2014; Warner, Tingle \& Kellet, 2013). Este sentimiento de comunidad, está respaldado por la literatura deportiva general, que postula el deporte como uno de los pocos escenarios que fomenta un sentido de comunidad en las personas (Nordstrom, Warner \& Barner; 2016; Warner \& Dixon, 2011; Warner, Tingle \& Kellett, 2013).

En relación al ámbito familiar, la conciliación de la vida laboral con la familiar es uno de los conflictos más destacados por las árbitras a la hora de establecer las razones por las cuales abandonaron el arbitraje. En esta línea, y de acuerdo con Perreau-Niel y Erard (2012) y Ridinger, Warner, Tingle y Kim (2017), los viajes y el desarrollo de su actividad arbitral eran incompatibles con los horarios de trabajo y en ocasiones les provocaba conflictos con sus parejas. Igualmente, las árbitras que son madres les puede dificultar la labor del arbitraje se destaca que la maternidad supone un escollo más en la conciliación de la crianza de niños y la labor de arbitraje (Kim \& Hong, 2016). No obstante, se ha demostrado que el apoyo de las federaciones resulta vital para reducir las dificultades en esta dimensión (Cuskelly \& Hoye, 2013; Hong, Jeong \& Downward, 2019; Kim, 2017).

En lo que al ámbito institucional se refiere, históricamente, gran parte de la estructura, instituciones y prácticas en el ámbito del fútbol, se han basado en normas patriarcales que han excluido a las mujeres del fútbol durante mucho tiempo (Scraton, Caudwell \& Holland, 2005). A lo largo de los siglos, la práctica del deporte y su organización se ha distinguido en función del sexo, habiendo deportes masculinos y deportes femeninos. (Jones \& Edwards, 2013).

Pero esta situación está cambiando, ya que se ha demostrado que el apoyo institucional percibido hace que las árbitras se sientan más relacionadas con la organización (Wu \& Liu, 2014) y, por ende, es probable que se sientan más autónomas en su trabajo. Este apoyo apreciado también puede proporcionar a las árbitras los recursos necesarios para realizar su trabajo de manera más eficiente y hacerlas sentir más competentes e incrementar la motivación interna (Gagné \& Deci, 2005; Mitchell, Gagné, Beaudry \& Dyer, 2012; Seibert \& Vallerand, 1997; Wang \& Courtright 2011).

En este sentido, la investigación de Kim y Hong (2016) indicaba que la contribución del apoyo institucional es uno de los factores que pueden paliar las consecuencias negativas del estrés e inspirar una actitud positiva respecto al desempeño laboral y de la intención de continuar trabajando en el sector, al detectarse como una de las causas de abandono. En esta línea, un estudio realizado Pérez-Muñoz et al. (2018) en España en categorías inferiores, las futbolistas afirmaban que jugaban al fútbol porque les gustaba, se divertían y tenían altos niveles de motivación para asistir a los entrenamientos.

En la misma línea, la investigación de Gagné, Forest, Gilbert, Aubé, Morin y Malorni (2010), mostró que el apoyo organizativo percibido estaba fuerte y positivamente vinculado a la motivación interna. Los resultados de este estudio también son consistentes con los del 
estudio de Tremblay, Blanchard, Taylor, Pelletier y Villeneuve (2009), quienes demostraron que el apoyo organizacional percibido predijo positivamente la motivación interna y destacó la importancia de los factores sociales como un determinante motivacional (Hong, Jeong \& Downward, 2019).

Por ello, la motivación interna es la mediadora entre el apoyo organizacional percibido y el conflicto de las árbitras entre el trabajo y la familia. El apoyo institucional percibido no es suficiente por sí solo, pero puede reducir los conflictos entre el trabajo y la familia a través de la motivación intrínseca (Hong, Jeong \& Downward, 2019), la cual, según Amorose (2007), puede aumentar a través de un clima motivacional positivo, ya que las árbitras en ese entorno tienden a invertir en la actividad más por su propio bien que como un medio para un fin. Por tanto, las motivaciones referidas al mantenimiento de la actividad, pasan necesariamente por una motivación intrínseca y el disfrute del fútbol en general y de la actividad del arbitraje en concreto, resaltando como factor clave las relaciones interpersonales y la existencia de un buen ambiente (Alonso-Arbiol, Arratibel \& Gómez, 2008).

Finalmente, la valoración por el ente institucional se convierte también en un aliciente que puede reforzar la motivación personal de las árbitras. Conjuntamente, percibir una valoración positiva por parte del organismo al que pertenecen puede provocar un alto nivel de motivación interna dando lugar a una mayor autodeterminación en su función de arbitraje (Hong, Jeong \& Downward, 2019).

Atendiendo a lo anteriormente expuesto, y siguiendo a Valenti, Scelles y Morrow (2018), son necesarias las contribuciones acerca de la experiencia de las mujeres en roles diferentes a los de jugadora, como es el caso de las árbitras, ya que no se le ha prestado la suficiente atención en la literatura existente. El estudio y la implementación de políticas favorables para ellas, mejorarían su nivel de empoderamiento, disminuyendo la brecha de género, y generando un poder productivo para abordar las tradicionales desigualdades de género (Kim \& Hong, 2016). Por ello, el objetivo del presente estudio es diseñar y validar una herramienta de evaluación de las barreras percibidas en el arbitraje femenino, tanto durante la formación de las árbitras (de manera previa), como una vez estas alcanzan este grado. De igual modo se pretende, a través de los resultados obtenidos, analizar las principales dificultades percibidas por las árbitras para el desempeño de sus funciones en el ámbito territorial de España.

\section{Material y método}

El presente trabajo se enmarca dentro de la investigación cuantitativa, a través de un estudio descriptivo y transversal, con la utilización de la encuesta como método de obtención de datos.

\section{Participantes}

La muestra de la presente investigación se obtuvo a través de un muestreo no probabilístico por conveniencia, resultando un total de 380 árbitras y árbitras asistentes de las diferentes categorías tanto nacionales como territoriales. Respecto a sus características, el 90\% tiene una edad de entre 18 a 34 años, la mayoría (91\%) no tienen a su cargo ninguna persona, el $75 \%$ vive sin pareja y el 39.8\% lleva entre 1 a 3 años arbitrando, mientras que el resto lleva en estas funciones más de 3 años.

\section{Instrumento}

Todas las escalas utilizadas en el cuestionario (escala 7 puntos Likert) fueron tomadas de la literatura y adaptadas al contexto de la investigación actual. La escala usada para la percepción de las barreras en el arbitraje femenino en fútbol por parte de las árbitras está compuesta por 31 ítems agrupados que tratan de dilucidar la percepción de las árbitras españolas de fútbol sobre las dificultades añadidas para desarrollar su trabajo por su condición de mujer. Estos ítems se encuentran agrupados en tres dimensiones en torno a las cuales se ha estructurado la percepción de las barreras: Percepción del Apoyo Institucional, Beneficios del Arbitraje y Percepción del Clima Social y Familiar.

La dimensión Percepción del Apoyo Institucional ha sido definida como la percepción del sustento de las instituciones hacia el arbitraje femenino en fútbol con un total de 10 ítems (Hong, Jeong \& Downward, 2019; Kim \& Hong, 2016). La dimensión Beneficios del Arbitraje presenta seis indicadores; en ella se pretende valorar la percepción de las arbitras sobre qué les aporta en su vida la labor arbitral (Hong, Jeong \& Downward, 2019; Kim \& Hong, 2016). La dimensión Percepción del Clima Social y Familiar presenta dos subdimensiones: por un lado, el ámbito social definido como la percepción del clima social ante el arbitraje femenino, distribuido en torno a seis atributos o ítems y por otro lado el ámbito familiar con nueve ítems, entendida como la percepción del clima familiar como una barrera que pueda dificultar el desempeño de su trabajo como árbitra (Hong, Jeong \& Downward, 2019; Kim \& Hong, 2016).

Antes de llegar al cuestionario definitivo, se com- 
probó la validez de contenido de la versión inicial a través de la evaluación por parte de un comité de expertos compuesto por 8 profesionales relacionados con el arbitraje y la gestión deportiva. Estos expertos revisaron la claridad, confiabilidad y el contenido de los ítems para evitar confusiones. Tras sus sugerencias, se realizaron algunas modificaciones en su redacción hasta llegar al cuestionario definitivo que fue suministrado a las árbitras de fútbol.

\section{Procedimiento}

El instrumento de medida se suministró a nivel nacional utilizando diferentes momentos y ubicaciones durante el 2019 y 2020. Los datos se recogieron por medios virtuales (cuestionario on-line). La herramienta se pasó a una muestra de árbitras y árbitras asistentes de las diferentes categorías tanto nacionales como territoriales $(\mathrm{n}=380)$, se contactó a con las árbitras a través del comité técnico de árbitros de la Real Federación Española de Fútbol.

\section{Análisis de datos}

Los datos fueron analizados mediante dos paquetes estadísticos, en primer lugar, mediante el software SPSS 21. IBM, Chicago y posteriormente mediante el software EQS 6.3, California. Se realizó un Análisis Factorial Exploratorio (AFE) (validez de constructo) mediante el método de componentes principales Varimax. Esto se llevó a cabo a través de la prueba de adecuación muestral Kaiser-Meyer-Olkin (KMO) y la prueba de esfericidad de Barlett (Zubillaga \& Cañadas,2021). Se obviaron aquellas cargas inferiores a 40 (Balanza, Morales, Guerrero \& Conesa, 2008; Calabuig, Mundina \& Crespo, 2010; Nunally, 1978; Wiersma, 2001), si bien se valoró mantener los ítems cercanos .40 a expensas de posteriores análisis. Para el análisis de la fiabilidad de la escala propuesta se calculó el á de Cronbach con valores superiores a .70 (Hair, Black, Babin, Anderson \& Tatham, 2006; Thomas \& Nelson, 2007). De esta manera nos aseguramos la estabilidad y una elevada consistencia interna de la escala.

Posteriormente se procedió al Análisis Factorial Confirmatorio (AFC) de primer orden y un posterior de segundo orden para constatar que tras las tres dimensiones existe un concepto superior o Percepción de las Barreras en el Arbitraje Femenino. Uno de los requisitos previos para el análisis de un modelo estructural es la confirmación de que las variables o constructos latentes están siendo medidos de forma adecuada, por lo que es necesario testar los modelos de medición
(Bagozzi \& Yi, 1991). Así, para el modelo propuesto, se comprueba la medición de la percepción de las árbitras en el desempeño de su tarea y teniendo en cuenta su condición de mujer. Para evaluar la adecuación de este modelo, se utilizan los siguientes índices de ajuste: Bentler-Bonnet Non-Normed Fit Index (NNFI), Comparative Fit Index (CFI), Standardzed Root Mean Square Residual (SRMR), Root Mean Square Error Approximation (RMSEA). El adecuado ajuste del modelo vendrá indicado por valores superiores a .90 en los índices IFI y CFI e inferiores a .05 en los índices SRMR y RMSEA. Si bien diferentes autores, en relación con el índice RMSEA, señalan como aceptables valores inferiores a .08 (Alvarado-Herrera, Bigné, Aldas-Manzano \& Curras-Pérez, 2015; Kim, Jun,Walker \& Drane, 2015; Kim \& Walker, 2012; Lorde, Greenidge \& Devonish, 2011; Ma \& Kaplanidou, 2016; Ma, Ma, Wu \& Rotherham, 2013; Parra, Elasri,Triadó \& Aparicio, 2016; Parra, González, Añó \& Ayora, 2016; Walker \& Kent, 2009).

Para la descripción de los resultados se utilizaron los estadísticos descriptivos siguientes: media, desviación típica, asimetría y curtosis de cada uno de los ítems.

\section{Resultados}

\section{Estadísticos descriptivos}

En las tablas 1a y $1 \mathrm{~b}$ se pueden observar los resultados de media, desviación, asimetría y curtosis de todos los indicadores que componen la escala en su fase inicial. Como se aprecia, todas las dimensiones se encuentran en el lado positivo de la escala, en especial la dimensión Beneficios del Arbitraje con indicadores cercanos al valor máximo de la escala. Las otras dos dimensiones, si bien están orientadas hacia el lado positivo de la escala, se encuentran cerca del valor neutral (4 «ni de acuerdo / ni en desacuerdo»).

$\mathrm{Al}$ analizar la escala por ítems se aprecia que dentro de la dimensión Percepción del Apoyo Institucional hay dos indicadores con valores en la respuesta de alto de acuerdo, en concreto son: «La institución es comprensiva ante mis ausencias por enfermedad y/o lesión" ( $\mathrm{M}=5.52$; $\mathrm{DT}=1.21)$ y "Cuando tengo un problema la institución me ayuda» $(\mathrm{M}=5.29 ; \mathrm{DT}=1.65)$ y destaca la presencia del único indicador en el lado negativo de la escala «Cuando necesito algo especial la institución está dispuesta a ayudarme»" $(\mathrm{M}=3.28 ; \mathrm{DT}=1.67)$. Al analizar la segunda dimensión Beneficios del Arbitraje se aprecia que es la dimensión que presenta los indicadores más cercanos a los valores máximos positivos de la escala, en especial «El arbitraje 
me enseña cosas como persona», con el valor más alto $(\mathrm{M}=6.56 ; \mathrm{DT}=.89)$ seguido de $«$ Ser arbitra me hace sentirme bien como persona» $(\mathrm{M}=6.36 ; \mathrm{DT}=.97) \mathrm{y}$ «El arbitraje me permite conocerme a mí misma (mis debilidades, fortalezas)» $(\mathrm{M}=6.36 ; \mathrm{DT}=.89)$. La última dimensión Percepción del Clima Social y Familiar es la que presenta una mayor dispersión en la valoración de los ítems; para facilitar su comprensión se debe analizar por separado el clima social del familiar. Los ítems de la percepción del clima social están todos formulados en sentido negativo, hay dos ítems que se encuentran en lado más bajo de la escala, son «El arbitraje femenino está subestimado por la sociedad en general» $(\mathrm{M}=2.46 ; \mathrm{DT}=1.42) \mathrm{y}$ «El arbitraje femenino está constantemente criticado» $(\mathrm{M}=2.71$; $\mathrm{DT}=1.53)$ es decir las árbitras en estos dos ítems no están de acuerdo con que la sociedad tenga una consideración negativa de su labor como árbitras. En cambio, los otros tres indicadores están ligeramente orientados hacia al «más bien de acuerdo» sin llegar a él, lo que nos apunta hacia una percepción negativa en estos indicadores.

$\mathrm{Al}$ analizar los ítems relacionados con la percepción del Clima familiar se detecta que casi todos tienen una consideración negativa; en el caso de los que están formulados en sentido negativo es «Debido a mis obligaciones familiares no finalizo mis tareas como árbitra» el que tiene un mayor valor $(\mathrm{M}=5.77$; $\mathrm{DT}=1.40)$. En el otro extremo es «El arbitraje interfiere en mi vida familiar el que presenta el valor más bajo» $(\mathrm{M}=3.61$; $\mathrm{DT}=1.96)$. Por último, se puede observar que todos los valores de asimetría y curtosis presentan valores aceptables que ya que están muy por debajo de 3.0 (Bentler, 1995).

Tabla 1a

\begin{tabular}{|c|c|c|c|c|c|}
\hline & Ítem & $M$ & DT & $\mathbf{A}$ & $\mathrm{C}$ \\
\hline $\mathrm{N}^{\circ}$ & \multicolumn{5}{|l|}{ Percepción del Apoyo Institucional (AI) } \\
\hline AI1. & La institución valora mi contribución al arbitraje & 5.04 & 1.55 & -.87 & .261 \\
\hline AI2. & La institución tiene en cuenta mis objetivos y forma de trabajar & 4.71 & 1.56 & -.58 & -.13 \\
\hline Al3. & $\begin{array}{l}\text { La institución es comprensiva ante mis ausencias por } \\
\text { enfermedad y/o lesión }\end{array}$ & 5.52 & 1.70 & -1.21 & .67 \\
\hline AI4. & Cuando tengo un problema la institución me ayuda & 5.29 & 1.65 & -1.02 & .34 \\
\hline Al5. & La institución se ocupa por mi bienestar & 4.95 & 1,71 & -.71 & -.29 \\
\hline Al6. & $\begin{array}{l}\text { Cuando necesito algo especial la institución está dispuesta a } \\
\text { ayudarme }\end{array}$ & 3.28 & 1.67 & .64 & -.32 \\
\hline Al7. & La institución se preocupa porque esté satisfecha & 4.77 & 1.71 & -.65 & -.35 \\
\hline AI8. & La institución tiene en cuenta mi opinión & 4.44 & 1.74 & -.50 & -.62 \\
\hline Al9. & La institución está satisfecha con mi trabajo como arbitra & 5.07 & 1.27 & -.90 & 1.13 \\
\hline \multirow[t]{2}{*}{ Al10. } & $\begin{array}{l}\text { La institución se esfuerza porque esté satisfecha con mi trabajo } \\
\text { como arbitra }\end{array}$ & 4.87 & 1.67 & -.73 & -.22 \\
\hline & \multicolumn{5}{|l|}{ Beneficios del Arbitraje (BA) } \\
\hline BA1. & Ser arbitra me hace sentirme bien como persona & 6.36 & .97 & -1.72 & 2.79 \\
\hline BA2. & El arbitraje aumenta mi autoestima & 5.87 & 1.28 & -1.46 & 2.22 \\
\hline BA3. & El arbitraje me enseña cosas como persona & 6.56 & .71 & -1.58 & 1.91 \\
\hline BA4. & Soy arbitra porque considero que es algo importante & 5.62 & 1.49 & -1.21 & 1.04 \\
\hline BA5. & Ser arbitra me hace pensar bien de mí misma & 5.94 & 1.18 & -1.42 & 2.65 \\
\hline BA6. & $\begin{array}{l}\text { El arbitraje me permite conocerme a mí misma (mis } \\
\text { debilidades, fortalezas) }\end{array}$ & 6.36 & .89 & -1.92 & 2.50 \\
\hline
\end{tabular}

\section{Análisis factorial exploratorio}

Antes de la estimación del modelo estructural, fue necesario evaluar las propiedades psicométricas de las

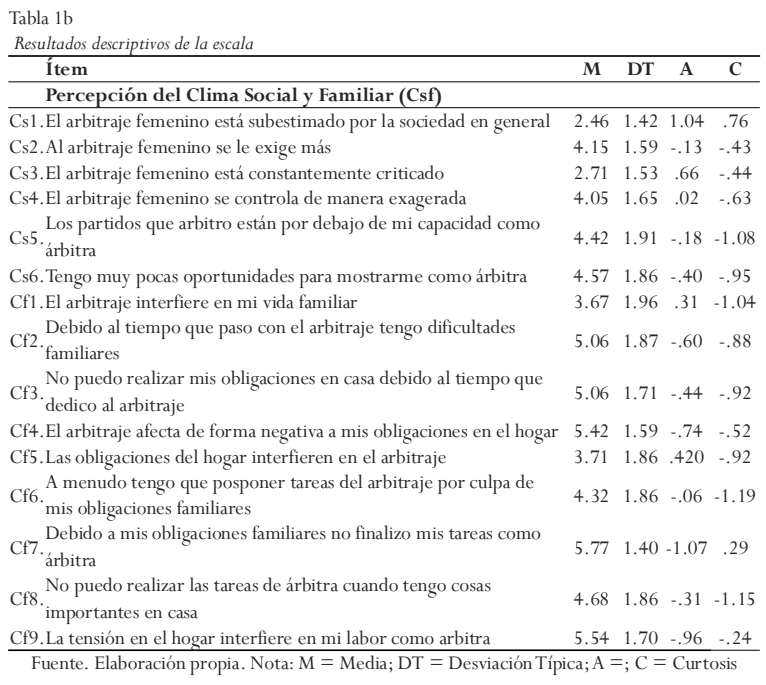

escalas. Para conseguir este objetivo se realiza un AFE (validez de constructo) mediante el método de componentes principales Varimax. Esto se llevó a cabo a través de la prueba de adecuación muestral Kaiser-MeyerOlkin $(\mathrm{KMO}=.887$ ) y la prueba de esfericidad de Barlett $\left(\mathrm{X}^{2}=6225.239\right.$; gl. $\left.=496 ; \mathrm{p}<.00\right)$. Una vez realizada la rotación de los factores, los ítems se agruparon en tres dimensiones. Se obviaron aquellas cargas inferiores a .40 , en concreto se eliminaron cuatro ítems de la dimensión Percepción del Clima Social y Familiar (Cs1, Cs3, Cf1, Cf6, Cf9), todos ellos con cargas factoriales inferiores a .40 . La práctica totalidad de las variables saturan un único factor que se corresponde con la dimensionalidad propuesta. La distribución de los ítems resulta lógica. Dado el resultado de los datos obtenidos, se podría interpretar la validez factorial.

Para el análisis de la fiabilidad de la escala propuesta se calculó el á de Cronbach. Las correlaciones resultantes fueron superiores a lo recomendado por diferentes autores (Sánchez-Pato; Palderón; Arias-Estero; GarcíaRoca; Bada \& Meroño et al., 2016; Thomas \& Nelson, 2007), alcanzándose un valor de 0.823 . Respecto a cada una de las dimensiones establecidas, el factor Percepción del Apoyo Institucional alcanzó un á de Cronbach de .864 , el factor Beneficios del Arbitraje un $.810 \mathrm{y} \mathrm{el}$ factor Percepción del Clima Social y Familiar un .744, siendo esta dimensión la que presenta el valor más débil. De esta manera se aseguró la estabilidad y una elevada consistencia interna de la escala.

\section{Análisis factorial confirmatorio}

Tras la obtención de estos datos, se procedió al AFC de primer orden con varias etapas y posteriormente, a un análisis de segundo orden para constatar que tras las tres dimensiones y los 27 ítems existe un concepto superior o Percepción de las Barreras en el Arbitraje Fe- 
menino. La figura 1 muestra el modelo final obtenido tras varios ajustes compuesto por tres dimensiones y 20 ítems, en este modelo final se eliminaron un total de siete ítems, uno de la dimensión Beneficios del Arbitraje (BA4) y seis de la dimensión Percepción Clima Social y Familiar (Cs2, Cs4, Cs5, Cs6 y Cf3). Además, se correlacionaron los errores de medida de varios ítems (BA1, AF1, AF6, AF5, Cf2 y Cf4). Los índices del AFC tras estas modificaciones muestran que el modelo desde la $1^{\text {a }}$ etapa, con índices inaceptables, hasta llegar a la $3^{\mathrm{a}}$ se ajusta de forma correcta a los datos (Byrne, 2008): $1^{\mathrm{a}}$ etapa $\mathrm{S}-\mathrm{B} \div{ }^{2}(461)=785.600 \mathrm{p}<.000 ; * \mathrm{CFI}=.52$; $*$ IFI $=.54, *$ RMSEA $(90 \%$ CI $)=.125(0.109-0.138)$; $\mathrm{SRMR}=.128 .3^{\mathrm{a}}$ etapa $\mathrm{S}-\mathrm{B} \div{ }^{2}(161)=/ 186.037, \mathrm{p}<$. $001 ; * \mathrm{CFI}=.94 ; * \mathrm{IFI}=.94, * \mathrm{RMSEA}(90 \% \mathrm{CI})=.059$ (0.000-0.092); SRMR $=.058$. Los resultados confirman la existencia de los tres factores propuestos inicialmente: Percepción del Apoyo Institucional, Beneficios del Arbitraje y Percepción del Clima Social y Familiar. En la figura 1 se puede observar el modelo final del AFC de primer orden con los pesos factoriales de cada atributo, las covarianzas entre factores y la varianza explicada.

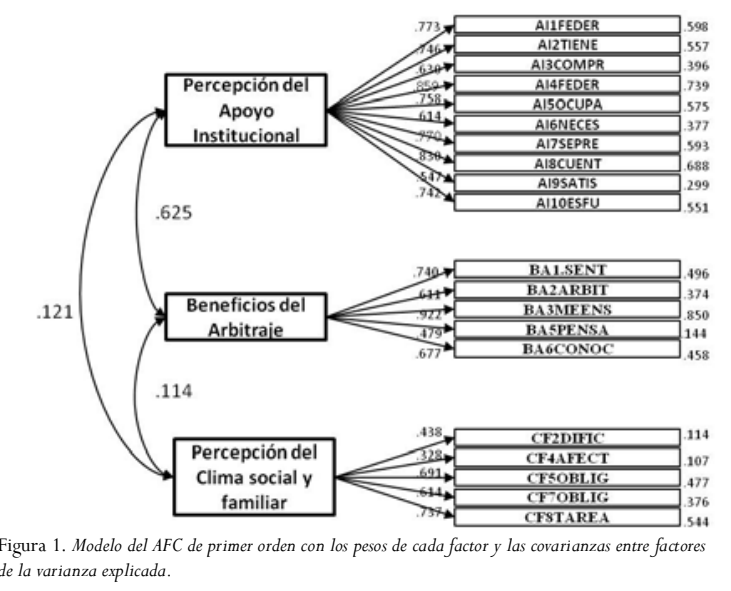

El análisis factorial de segundo orden confirma el modelo estructural propuesto para la percepción de las barreras en el arbitraje femenino en fútbol (figura 2). Como se puede observar los coeficientes â resultantes de la solución estandarizada permiten decir que todos los factores de Percepción del Apoyo Institucional y Beneficios del Arbitraje tienen un impacto positivo y significativo sobre la percepción de las barreras por parte de las árbitras de fútbol, mientras que la dimensión Percepción Clima Social y Familiar tiene un impacto negativo y significativo. Se observa como el factor de Beneficios del Arbitraje es el que presenta un mayor impacto $(\hat{\mathrm{a}}=.815)$ sobre la percepción de las barreras en el arbitraje femenino. El segundo factor por importancia en impacto es la Percepción del Apoyo Institucional ( $\hat{a}=$ .767). El que menor impacto tiene sobre la percepción de las barreras, en sentido negativo, pero es igualmente significativo, es el factor Percepción del Clima Social y Familiar ( $\hat{a}=-.148)$.

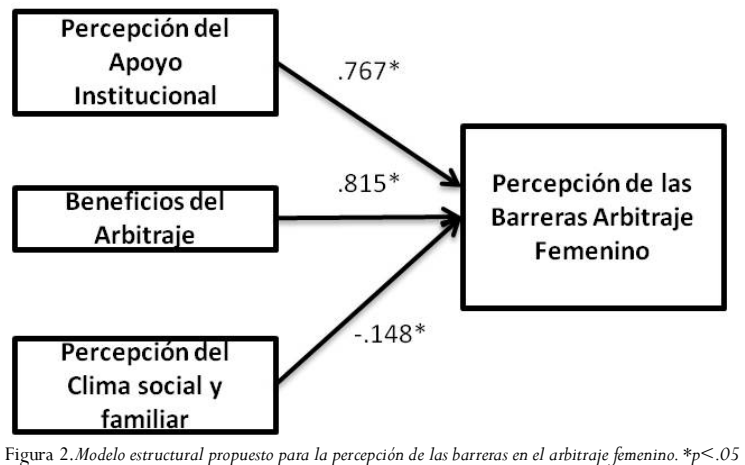

\section{Discusión}

El objetivo principal del presente estudio, fue diseñar y validar una herramienta de evaluación de las barreras percibidas en el arbitraje femenino, al no encontrar en la literatura analizada un cuestionario válido y fiable que aunara las diferentes dimensiones implicadas en la percepción de las barreras sociales, familiares, institucionales y motivacionales de este colectivo (Amorose, 2007; Cuskelly \& Hoye, 2013; Hong, Jeong \& Downward, 2019; Kim, 2017; Kim \& Hong, 2016; Logan, 2012; Mitchell, Gagné, Beaudry \& Dyer, 2012; Ridinger, Warner, Tingle \& Kim, 2017; Seibert, Wang \& Courtright, 2011;Tingle,Warner \& Sartore-baldwin, 2014; Wu \& Liu, 2014).

Una vez analizados y adaptados al contexto los ítems atendiendo a las investigaciones previas de referencia, se procedió a evaluar la validez de contenido a través de un comité de expertos, para la revisión de la claridad, confiabilidad y el contenido de los ítems, procediendo a las modificaciones sugeridas. Estos se agruparon en tres dimensiones principales: Percepción del Clima Social y Familiar, Percepción del Apoyo Institucional y Beneficios del Arbitraje, relacionada con los aspectos motivacionales (Hong, Jeong \& Downward, 2019; Kim \& Hong, 2016).

Los resultados de las pruebas realizadas confirman la validez y fiabilidad de la presente escala para la población objeto de estudio. La validez de constructo se analizó a través del análisis factorial exploratorio, cuyos resultados mostraron que la práctica totalidad de las variables saturan un único factor que se corresponde con la dimensionalidad propuesta, percibiéndose una distribución lógica de los ítems. Del mismo modo, se garantizó la estabilidad y una elevada consistencia in- 
terna de la escala a través del á de Cronbach, cuyas correlaciones fueron superiores a lo recomendado por diferentes autores (Sánchez-Pato; Palderón; Arias-Estero; García-Roca; Bada \& Meroño et al., 2016; Thomas \& Nelson, 2007), alcanzándose un valor de 0.823. Por último, los resultados obtenidos en el análisis factorial confirmatorio de primer y segundo orden, constatan que tras las tres dimensiones existe un concepto superior o Percepción de las Barreras en el Arbitraje Femenino, confirmando el modelo estructural y la existencia de los tres factores propuestos inicialmente: Percepción del Apoyo Institucional, Beneficios del Arbitraje y Percepción del Clima Social y Familiar.

Respecto al objetivo secundario del presente estudio, se ha analizado la percepción del colectivo arbitral femenino, para determinar cuáles son las verdaderas barreras y dificultades que se encuentran, a través del análisis de 380 cuestionarios de árbitras de fútbol, estructurado en las tres dimensiones mencionadas anteriormente.

Referente a la dimensión de la Percepción del Apoyo Institucional, los resultados obtenidos evidencian que las árbitras están muy de acuerdo en que la institución es comprensible ante las ausencias por enfermedad y/o lesión y las árbitras consideran que cuando tienen un problema la institución les ayuda.

Los resultados obtenidos relacionados con la percepción del apoyo Institucional van en consonancia con el estudio de Azurmendi (2016), en el que las árbitras se sienten apoyadas por parte de la institución. Por el contrario, en cuanto a la retribución económica, las árbitras entrevistadas muestran un menor acuerdo con la organización, ya que la retribución es menor en las categorías elevadas en comparación a las competiciones profesionales en el fútbol masculino.

Por el contrario, estos resultados coinciden con los obtenidos cuando se les pregunta acerca de si cuando necesitan algo especial, la institución está dispuesta a ayudarme. En este sentido, un estudio realizado por Demers y Audet (2007), las árbitras dicen que, sí reciben un apoyo especial dependiendo de la experiencia de las árbitras, las más jóvenes el apoyo recibido es técnico y las árbitras más experimentadas reciben apoyo por parte de la institución para afrontar obstáculos por ser mujer.

Al analizar la segunda dimensión de Beneficios del Arbitraje se aprecia que es aquella que presenta los indicadores más cercanos a los valores máximos positivos de la escala. El indicador mejor valorado, se refiere a si el arbitraje les enseña cosas como persona. Por otro lado, también se obtiene una valoración muy buena en los indicadores que hacen referencia a que el ser árbitra les haga sentir bien como persona y les permita conocerse a sí mismas en cuanto a debilidades y fortalezas.

En esta dimensión, las árbitras consideran que arbitrar aumenta su autoestima, en la línea de los resultados obtenidos por Azurmendi (2106), el cual afirma que las árbitras manifiestan haberse sentido rodeadas de compañeros que les han ayudado y apoyado tanto en el inicio como en situaciones de desánimo durante el desempeño de su rol y con la consiguiente mejora de su autoestima. Asimismo, estas mujeres trasmiten sentirse muy apoyadas por su familia, pareja o grupo de amistades. Por ello, la mayoría de las entrevistadas no prevén abandonar el arbitraje. En este sentido, algunas árbitras desearían continuar y ascender de nivel, incluso llegar a ser internacionales, sin embargo, otras manifiestan estar a gusto como están. Estos resultados corroboran los del presente estudio y evidencian que, en líneas generales, el arbitraje les aporta muchos beneficios.

Referente a los beneficios del arbitraje, las árbitras consideran que es algo importante y les hace pensar bien en ellas mismas. Estos resultados poseen similitudes con el estudio realizado por Nordstrom, Warner y Barner (2016), resalta que las árbitras se sentían orgullosas cuando arbitraban, pudiendo de esta forma continuar siendo parte del juego, experimentando sensaciones similares de emoción y adrenalina que cuando jugaban.

La última dimensión de Percepción del Clima Social y Familiar es la que presenta una mayor dispersión en la valoración de los ítems. En cuanto a la Percepción del Clima Social, se puede resaltar la cuestión referida a si el arbitraje femenino está subestimado por la sociedad en general, es decir, las árbitras no están de acuerdo con que la sociedad tenga una consideración negativa de su labor como árbitras.

El deporte, y en particular el fútbol, es un campo de gran potencia para la construcción de una identidad masculina, siendo muy dominante en numerosos países y, por consiguiente, el elevado número de árbitras del fútbol femenino constituye una amenaza directa a la identidad masculina, creando un importante foco de conflicto de género (Mean, 2001). Los datos obtenidos son muy diferentes a los estudios encontrados, están vinculados a la opinión que tiene la sociedad del arbitraje femenino y van relacionados con comentarios sexistas. Las ofensas hacia las mujeres en el ámbito deportivo se materializan a través de este tipo de comentarios, que algunas autoras como Kerr (2009), o Azurmendi y Fontecha 
(2015) califican como acoso sexual en el deporte. Del mismo modo, también se aprecia en los resultados obtenidos de Forbes, Edwards, y Fleming (2015), una amplia gama de abusos dirigidos a las árbitras de fútbol femenino que, en general, podrían interpretarse como sexistas.

Cabe destacar que los resultados de la presente investigación coinciden con los obtenidos por Azurmendi (2016), en los que las árbitras en general se sienten respetadas por el entorno, aunque algunas reconocen que esta situación es resultado de una trayectoria en la que han tenido que demostrar que son capaces. Por el contrario, todo lo que rodea a las árbitras en la sociedad en general, provocó reticencias por parte del entorno familiar, sintiendo la necesidad de acompañarlas a los partidos que arbitraban.

En los resultados obtenidos relacionados con si consideran que tienen muy pocas oportunidades para mostrarse como árbitras, estos van en consonancia con los datos aportados por Azurmendi (2016), en los que se manifiestan que no han sido valoradas como sus homólogos hombres, y reconocen haber tenido que demostrar más para obtener el mismo reconocimiento que ellos.

En esta línea, cabe destacar que las participantes en el estudio tienen la percepción de sentirse menos valoradas por el entorno que los hombres (Norman, 2010), resultados que poseen similitudes con esta investigación. Estas coincidencias se trasladan a otros deportes, como en la investigación realizada por McGinnis, McQuillan y Chapple (2005) sobre las mujeres golfistas, en la que se evidenciaba que las mujeres en los deportes dominados por los hombres se sentían injustamente estereotipadas y bajo una mayor presión de rendimiento.

Otro indicador a resaltar es si consideran que en el arbitraje femenino se le exige más. En este sentido, una investigación llevada a cabo por Souchon, Cabagno, Traclet, Dosseville, Livingstone y Jones et al. (2010) con árbitros y árbitras de balonmano, puso de manifiesto que las decisiones arbitrales están condicionadas por los estereotipos; se presupone que las jugadoras son menos capaces que los hombres de continuar el juego una vez cometida una falta, considerándolas menos competentes en los deportes de contacto. El estudio aportado por Brake (2010) y Norman (2008) poseen similitudes con los anteriores autores, ambos corroborando que las árbitras perciben que en el entorno deportivo se cuestionan sus capacidades para desempeñar estos roles con solvencia.
En cuanto a la dimensión sobre la percepción del Clima familiar, el arbitraje es una actividad que exige una gran inversión de tiempo, y éste es un factor que desencadena conflictos familiares entre las mujeres que desean ser árbitras (Christensen, Guttman \& Pfister, 2001), ya que sienten que no pueden compatibilizar las exigencias de este rol con las responsabilidades familiares (Lavoi \& Dutove, 2012; Mccharles, 2010). Según las conclusiones alcanzadas por Azurmendi (2106), esto supone un obstáculo para las árbitras que viven en pareja o tienen hijos y, más si cabe, si la familia cuestiona su continuidad en el arbitraje.

En esta dimensión, si bien las árbitras encuestadas resaltan que, debido a sus obligaciones familiares, estas no finalizan sus tareas como árbitra, en el otro extremo presentando el valor más bajo, detallan que el arbitraje no interfiere en su vida familiar. Los resultados pueden interpretarse, siguiendo investigaciones previas de referencia (Azurmendi, 2016; Kerr \& Marshall, 2007; McCharles, 2010; Reade, Rodgers \& Norman, 2009), de forma que, para ellas, la familia constituye una prioridad en sus vidas, y condiciona el modo en que éstas se vinculan con la labor de arbitraje.

Asimismo, Gallego y Estebaranz (2005) subrayan que las árbitras deben conciliar el deporte con la pareja, la maternidad y otras actividades como los estudios o el trabajo. Según Kerr y Marshall (2007), el conflicto que viven estas mujeres se debe, sobre todo, a la división sexual de los roles, y adquiere especial relevancia cuando no existe corresponsabilidad en las tareas del hogar, debido a que sienten excesiva carga de responsabilidades y tareas domésticas que requieren dedicación, datos que coinciden totalmente con las árbitras analizadas, las cuales indican que el arbitraje afecta de forma negativa a las tareas del hogar.

\section{Conclusiones}

De manera general, las árbitras, sienten el apoyo de las instituciones ante diferentes problemas que les puedan surgir.

Las árbitras destacan que ejercer les aporta unos beneficios personales altos.

No consideran que la sociedad las menosprecie pero sí expresan las dificultades para conciliar la vida familiar y el arbitraje.

El establecimiento de medidas correctoras respecto a las principales barreras percibidas por las mujeres en el sector deportivo puede generar mejores condiciones en su desempeño profesional, igualmente, en 
la sociedad en general.

Resalta que las árbitras perciben grandes beneficios en la función de arbitraje que realizan, si bien se perciben numerosas barreras en todas las dimensiones analizadas, siendo necesario el establecimiento de políticas para facilitar el acceso de la mujer al arbitraje en fútbol.

La escala elaborada se presenta como una herramienta válida y fiable para determinar las principales barreras asociadas al arbitraje femenino.

\section{Implicaciones para la gestión y futuras lí- neas de investigación.}

Tras el análisis de las percepciones de las árbitras, el gestor/a deportivo/a podrá tener el conocimiento necesario para la realización y orientación de políticas y estrategias justas, equitativas e igualitarias, en los aspectos detectados como barreras, en un mundo tradicionalmente considerado exclusivo de los hombres.

Respecto a las futuras líneas de investigación, se plantea la necesidad de extrapolar el presente estudio al arbitraje de carácter internacional estableciendo un estudio cross cultural debido a la gran diferencia de derechos entre las mujeres en cada una de las naciones.

\section{Referencias}

Alonso-Arbiol, I., Arratibel, N. \& Gómez, E. (2008). La motivación del colectivo arbitral en fútbol: Un estudio cualitativo. Revista de Psicología del Deporte, (17)2, 187-203. Recuperado de https://www.redalyc.org/pdf/2351/ 235119254001.pdf.

Alvarado-Herrera, A., Bigné, E., Aldas-Manzano, J. \& CurrasPérez, R. (2015). A scale for measuring consumer perceptions of corporate social responsibility following the sustainable development paradigm. Journal of Business Ethics,(140)2, 243-262. Recuperado de https:// link.springer.com/content/pdf/10.1007/s10551015-2654-9.pdf.

Amorose, A. (2007). Coaching effectiveness: Exploring the relationship between coaching behavior and selfdetermined motivation. En Intrinsic motivation and selfdetermination in exercise and sport (pp. 209-227, 347-351). Champaign, IL: Human Kinetics.

Azurmendi, A. \& Fontecha, M. (2015). Guía para prevenir el acoso y el abuso sexual a mujeres en el deporte: Pautas para elaborar un protocolo.Vitoria-Gasteiz, PaísVasco: Emakunde.

Azurmendi, A. (2016). Obstáculos psicosociales para la participación de las mujeres en el deporte como entrenadoras y árbitras. Tesis doctoral. Universidad del País Vasco. Recuperado de https://addi.ehu.es/bitstream/handle/10810/
$26195 /$ TESIS_A Z U R M E N D I_E C H E GARAY_AINHOA.pdf?sequence $=1$ \&isAllowed $=\mathrm{y}$.

Balanza, S., Morales, I., Guerrero, J. \& Conesa, A. (2008). Fiabilidad y validez de un cuestionario para medir en estudiantes universitarios la asociación de la ansiedad y depresión con factores académicos y psicosociofamiliares durante el curso 2004-2005. Revista Española de Salud Pública, 82, 189-200. Recuperado de https:// www.scielosp.org/pdf/resp/2008.v82n2/189-200.

Brake, D. (2010). Getting in the game:Title IX and the women's sports revolution. NewYork, NY: NewYork University.

Calabuig, F., Mundina, J. \& Crespo, J. (2010). Eventqual: una medida de la calidad percibida por los espectadores de eventos deportivos. Retos. Nuevas Tendencias en la Educación Física, Deporte y Recreación, 18, 66-70. Recuperado de https : / / ww w.redalyc.org / pdf / 3457 / 345732284013.pdf.

Caudwell, J. (2011). Gender, feminism and football studies. Soccer \& Society, (12)3, 330-344. doi: https://doi.org/ 10.1080/14660970.2011.568099.

Christensen, K., Guttmann, A. \& Pfister, G. (2001). International encyclopaedia of women and sports. New York, NY: Macmillan.

Cleland, J., Pope, S. \& Williams, J. (2020). I do worry that football will become over-feminized: Ambiguities in fan reflections on the gender order in men's professional football in the United Kingdom. Sociology of Sport Journal, (37)4, 366-375. doi: https://doi.org/10.1123/ ssj.2019-0060.

Cuskelly, G. \& Hoye, R. (2013). Sport officials' intention to continue. Sport Management Review, (16)4, 451-464. doi: https: / / doi.org/10.1016/j.smr.2013.01.003.

Demers, G. \& Audet M. H. (2007). What we know about the experience of women beginner coaches. Canadian Journal forWomen in Coaching, (8)1, 1-8.

Flanagan, M.K. (2013). Managing coed soccer: Gender, power, and participation. Tesis doctoral. Florida State University. Recuperado de https://fsu.digital.flvc.org/islandora/ object/fsu\%3A183716/.

Gagné, M. \& Deci, E. (2005). Self-determination theory and work motivation. Journal of Organizational Behavior, (26)4, 331-362. doi: https://doi.org/10.1002/job. 322.

Gagné, M., Forest, J., Gilbert, M-H. Aubé, C; Morin, E. \& Malorni, Á. (2010). The motivation at work scale: Validation evidence in two languages. Educational and Psychological Measurement, (70)4, 628-646. doi: https:// doi.org/10.1177/0013164409355698.

Hair, J., Black,W., Babin, B., Anderson, R. \&Tatham, R. (2010). Multivariate Data Analysis. New Jersey: Pearson Prentice Hall.

Hong, E., Jeong, Y. \& Downward, P. (2019). Perceived organizational support, internal motivation, and workfamily conflict among soccer referees. Managing Sport and Leisure, (24)1-3, 141-154. doi: https: / / doi.org/10.1080/ 
23750472.2019 .1593049$.

https: / / doi.org/10.1260/174795408787186431.

Jones, C. \& Edwards, L.L. (2013). The woman in black:

Exposing sexist beliefs about female officials in elite men's football. Sport, Ethics and Philosophy, (7)2, 202-216. doi: https: / / doi.org/10.1080/17511321.2013.777771.

Jones, K. (2008). Female fandom: Identity, sexism and men's professional football in England. Sociology of Sport Journal, (25)4, 516-537. doi: https://doi.org/10.1123/ ssj.25.4.516.

Kellett, P. \& Shilbury, D. (2007). Umpire participation: is abuse really the issue? Sport Management Review, (10)3, 209 229. doi: https://doi.org/10.1016/S1441 3523(07)70012-8.

Kellett, P. \& Warner, S. (2011). Creating communities that lead to retention: The social worlds and communities of umpires. European Sport Management Quarterly, (11)5, 471494. doi: https://doi.org/10.1080/ 16184742.2011.624109.

Kerr, G. \& Marshall, D. (2007). Shifting the culture: implications for female coaches. Canadian Journal forWomen in Coaching, (7)4, 1-4.

Kerr, G. (2009). Female coaches' experience of harassment and bullying. En taking the lead, strategies and solutions from female coaches. (pp. 57-72) .Canada: The Univertity of Alberta press.

Kim, M.C. \& Hong, E. (2016). A red card for women: Female officials ostracized in South Korean football. Asian Journal ofWomen's Studies, (22)2, 114-130. doi: https: / / doi.org/ 10.1080/12259276.2016.1168156.

Kim, S. (2017). Perceived organizational support as a mediator between distributive justice and sports referees' job satisfaction and career commitment. Annals of leisure research, (20)2, 169-187. doi: https: / / doi.org/10.1080/ 11745398.2016.1147363.

Kim,W., Jun, H.M.,Walker, M. \& Drane, D. (2015). Evaluating the perceived social impacts of hosting large-scale sport tourism events: scale development and validation. Tourism management, 48, 21-32. doi: https: / / doi.org/10.1016/ j.tourman.2014.10.015.

lac.gc.ca/.item ?id=NR67694\&op=pdf\&app $=$ Library\&oclc_number $=796917556$

Lavoi, N. \& Dutove, J. (2012). Barriers and supports for female coaches: an ecological model. Sports Coaching Review, (1)1, 17-37. doi: https://doi.org/10.1080/ 21640629.2012.695891.

Logan, G. (2012). Some of what we discovered was shocking. it is discrimination. Recuperado el 24 de marzo de 2020, de: http:/ /www.bbc.com/sport/0/football/17598719.

Lorde, T., Greenidge, D. \& Devonish, D. (2011). Local residents' perceptions of the impacts of the icc cricket world cup 2007 on barbados: comparisons of pre- and post-games. Tourism management, (32)2, 349-356. doi: https://doi.org/10.1016/j.tourman.2010.03.004.
Ma, S.C., Ma, S.M., Wu, J.H. \& Rotherham, I. (2013). Host residents' perception changes on major sport events. European sport management quarterly, (13)5, 511-536. doi: https: / /doi.org/10.1080/16184742.2013.838980.

Mata, P. (2010). Árbitra no me ha llamado nadie:La situación de la mujer en el arbitraje de fútbol de Cantabria. Tesis de máster. Universidad de Cantabria. 2010.

Mccharles, B. (2010). Life stories of women in coaching. Tesis doctoral. University of Toronto. Recuperado de https: / / central.bac-

Mcginnis, L., Mcquillan, J. \& Chapple, C. (2005). I just want to play: Women, sexism and persistence in golf. Journal of Sport and Social Issues, (29)3, 313-37.doi: https: / / doi.org/ $10.1177 / 0193723504272659$.

Mean, L. (2011). Identity and discursive practice: Doing gender on the football pitch. Discourse \& Society, (12)6, 789-815. doi: https://doi.org/10.1177/ 0957926501012006004.

Mitchell, J., Gagné, M., Beaudry, A. \& Dyer, L. (2012). The role of perceived organizational support, distributive justice and motivation in reactions to new information technology. Computers in Human Behavior, (28)2, 729-738. doi: https: / /doi.org/10.1016/j.chb.2011.11.021.

Muller, J. (2014). Local Relations and Transnational Imaginaries: Football Practices of Migrant men andWomen From Andean Countries in Spain. Soccer \& Society, (15)4, 596-617. doi: https://doi.org/10.1080/ 14660970.2013.828601.

Nordstrom, H., Warner, S. \& Barner, J. (2016). Behing the Stripes: Female football officials experiences. Intertational Journal of Sport management and Marketing, 16, 259-279.

Norman, L. (2008). The UK coaching system is failing women coaches. International Journal of Sports Sciences \& Coaching, (3)4, 447-464. doi:

Norman, L. (2010). Feeling second best: Elite women coaches' experiences. Sociology of Sport Journal, 27, 89-104.

Nunally, J. (1978). Psychometric theory. NewYork, NY: McGrawHill.

Parra, D., Elarsi, A., Triadó, X. \& Aparicio, P. (2016). Análisis de la relación entre los beneficios y los costes percibidos y la satisfacción del residente con la celebración de un evento deportivo: efecto mediador del valor percibido. Revista de Psicología del Deporte, (25)3, 59-63. Recuperado de https://revistes.uab.cat/rpd/article/view/v25-n3parra-camacho-elasri-etal.

Parra, D., González, R., Año, V. \& Ayora, D. (2016). Percepción de los visitantes sobre el impacto social y sus intenciones con respecto a la celebración de un evento deportivo de pequeña escala. Revista de Psicología del Deporte, (25)3, 93-96. Recuperado de https: / / www.redalyc.org / pdf/2351/235146293022.pdf.

Pérez-Muñoz, S., Castaño, R., Sánchez-Muñoz, A., Rodríguez, A., De Mena, J.M., Fuentes, J.M y Castaño, C. (2018). ¿Por qué juego al fútbol si soy una mujer?: Motivaciones 
para jugar al fútbol. Retos. Nuevas Tendencias en la Educación Física, Deporte y Recreación, 34, 183-188. doi: https:// doi.org/10.47197/retos.v0i34.58572

Perreau-Niel,A. \& Erard, C. (2010). French football referees: An exploratory study of the conditions of access and employment for referees in terms of level and gender. Soccer \& Society, (16)1, 1-16 .doi: https://doi.org/ 10.1080/14660970.2012.627168.

Phillips, P. \& Fairley, S. (2014). Umpiring: a serious leisure choice. Journal of Leisure Research, (46)2, 84-202 .doi: https://doi.org/10.1080/00222216.2014.11950319.

Pope, S. (2013). The love of my life. The meaning and importance of sport for female fans. Journal of Sport and Social Issues, (37)2, 175-195. doi: https://doi.org/ $10.1177 / 0193723512455919$.

Reade, I., Rodgers, W. \& Norman, L. (2009). The underrepresentation of women in coaching: A comparison of male and female Canadian coaches at low and high levels of coaching. International Journal of Sports Science \& Coaching, (4)4, 505-520. doi: https://doi.org/10.1260/ 174795409790291439.

Reid, K. \& Dallaire, C. (2019). Because there Are So Few of Us: The Marginalization of Female Soccer Referees in Ontario, Canada. Women in Sport and Physical Activity Journal, (27)1, 12-20. doi: https://doi.org/10.1123/ wspaj.2017-0031.

Reid, K. \& Dallaire, C. (2020). I'd like to think I'm a good referee: Discourses of ability and the subjectivity of the female soccer referee in Ontario (Canada). Soccer \& Society, (21)7, 762-777. doi: https://doi.org/10.1080/ 14660970.2020.1742705.

Ridinger, L., Warner, S., Tingle, J. \& Kim, K. (2017). Why referees stay in the game. Global Sport Business Journal,(5)3, 22-37. Recuperado de https:// digitalcom mons.trinity.edu / c gi / viewcontent.cgi?article $=1053 \&$ context $=$ busadmin_faculty.

Sánchez-Pato,A., Calderón,A., Arias-Estero, J.L., García-Roca, J.A., Bada, J., Meroño, L., . . Sedgwick, M. (2016). Diseño y validación del cuestionario de percepción de los estudiantes universitarios-deportistas de alto nivel sobre la carrera dual (ESTPORT). Revista Cultura, Ciencia y Deporte, (11)32, 127-147. Recuperado de https:// www.redalyc.org/pdf/1630/163046240006.pdf.

Scraton, S., Caudwell, J. \& Holland, S. (2005). Bend it like Patel: Centering 'race', ethnicity and gender in feminist analysis of women's football in England. International Review for the Sociology of Sport, (40)1, 71-88. doi: https: / / doi.org/10.1177/1012690205052169.

Seibert, S., Wang, G. \& Courtright, S. (2011). Antecedents and consequences of psychological and team empowerment in organizations: A meta-analytic review. Journal of Applied Psychology, (96)5, 981-1003. Recuperado de https://psycnet.apa.org/buy/2011-06121-001.

Souchon, N., Cabagno, G., Traclet, A., Dosseville, F.,
Llivingstone, A., Jones, M. \& Maio, G. (2010). Referees' decision-making and player gender: The moderating role of the type of situation. Journal of Applied Sport Psychology, (22)1, 1-16. doi: https://doi.org/10.1080/ 10413200903250476.

Theberge, N. (1990). Gender, work, and power: the case of women in coaching. Canadian Journal of Sociology, 15, 5975. doi: https://doi.org/10.2307/3341173.

Thomas, J. \& Nelson, J. (2007). Métodos de investigación en actividad física. Les Guixeres, Cataluña: Editorial Paidotribo.

Tingle, J., Warner, S. \& Sartore-Baldwin, M. (2014). The experience of Former Women Officials and the Impact on the Sporting Community. Sex Roles, 71, 7-20. doi: https: / /doi.org/10.1007/s11199-014-0366-8.

Tremblay, M., Blanchard, C., Taylor, S., Pelletier, L. \& Villeneuve, M. (2009). Work extrinsic and intrinsic motivation scale: Its value for organizational research. Canadian Journal of Behavioural Science/Revue Canadienne des Sciences du Comportement, (41)4, 213-226. doi: https:/ /doi.org/10.1037/a0015167.

Valenti, M., Scelles, N. \& Morrow, S. (2018).Women's football studies: An integrative review. Sport, Business and Management, (8)5, 511-528. doi: https://doi.org/ 10.1108/SBM-09-2017-0048.

Vallerand, R. (1997). Toward a hierarchical model of intrinsic and extrinsic motivation. Advances in experimental social psychology, 29, 271-360. doi: https://doi.org/10.1016/ S0065-2601(08)60019-2.

Warner, S. \& Dixon, M. (2011). Understanding sense of community from the athlete's perspective. Journal of Sport Management, (259)3, 257-271.doi: https://doi.org/ 10.1123/jsm.25.3.257.

Warner, S., Tingle, J. \& Kellett, P. (2013). Ofûciating attrition: The experiences of former referees via a sport development lens. Journal of Sport Management, (27)4, 316 328. doi; https://doi.org/10.1123/jsm.27.4.316.

Wiersma, L. (2011). Conceptualization and development of the sources of enjoyment in youth sport questionnaire. Measurement in Physical Education and Exercise Science, (5)3, 153-177. doi: https://doi.org/10.1207/ S15327841MPEE0503_3.

Wu, C.C. \& Liu, N.T. (2014). Perceived organizational support, organizational commitment and service-oriented organizational citizenship behaviors. International Journal of Business and Information, (9)1, 61-88. Recuperado de https: / / search.proquest.com / openview / $47988 \mathrm{dfa} 1$ a 69 a e 19900746 fd 2609648 / 1 ?cbl=236248\&pq-origsite $=$ gscholar.

Zubillaga Olague, M., \& Cañadas, L. (2021). Diseño y validación del cuestionario «\#EvalEF» para conocer el proceso de evaluación desarrollado por los docentes de educación física. Retos, 42, 47-55. https: / / doi.org/10.47197/ retos.v42i0.86627 